\title{
Pediatric spinal dermoids - the silent legacy of dysraphism: Diagnosis, management and prognosis - a case series with review of literature
}

\begin{abstract}
Paediatric spinal dermoids are a subtype of cutaneous inclusion tumours of the spine consisting of ectodermal derivatives. They are relatively rare conditions with associated with the stigmata of spinal dysraphism with possible devastating consequences. We present a case series, in which we study the clinical, radiological and surgical aspects and follow up of the tumour in the last one year. All patients presented with either para or tetraplegia with or without sensory involvement. Contrast MRI was done in all cases, of which 3 were intradural extramedullary lesions and one was intramedullary lesion. The age ranged from 1 year to 4 years. 2 cases were associated with tethering of cord, 2 cases with dermal sinus and another with dandy walker malformation. One case had infected discharging sinus with dermoid. Gross total excision of tumour was done in all cases with Neuro monitoring. All had good post operative outcome without worsening of deficits. All patients received rehabilitation. Follow up study ranged from 3 months to 11 months. No residual tumour noted in follow up MRI.
\end{abstract}

Keywords: dermoid, epidermoid, spine, paediatric, intramedullary, intradural extramedullary, sinus tract
Volume II Issue 3 - 202 I

\author{
Bysani P, Ganapathy S, Adesh J, Pandya J, \\ Raykar R \\ Department of Neurosurgery, St. John's Medical Collage \\ Hospital, India
}

\begin{abstract}
Correspondence: Dr. Sibhi Ganapathy MS, Mch, DNB, MNAMS, FAGE, Assistant Professor, Department of Neurosurgery, St. John's Medical College \&amp; Hospital, Bangalore, India, Email sibhig09@gmail.com
\end{abstract}

Received: May 17, 2021 | Published: June 16, 2021

\section{Introduction}

Paediatric spinal dermoids are rare conditions with devastating consequences for the affected child. Few are diagnosed early despite the presence of stigmata of dysraphism (of which they can be associated with) The neurological compromise, especially to bowel and bladder control, can be irreversible leading to a lifetime of difficulty for the patient. ${ }^{1}$ Here we discuss a just a few interesting cases of dermoids of the paediatric spine operated at St. John's Hospital in Bangalore, south India over the past year.

\section{Case series}

\section{Case I}

A 1-year old male child presented with weakness of upper and lower limbs for 1 week. The weakness in lower limbs were much worse with inability to walk or stand. He was unable to sit by himself and had significant upper limb weakness as well with inability to grip objects for 1 month but worse since a week. On examination he was irritable, awake and consolable with a discharging sinus from the posterior aspect of the neck in the midline. NO evidence of inflammation or fever was seen. On examination of the nervous system his motor system revealed bilateral lower limbs hypertonia (Modified Ashworth score 4) while the upper limb tone was normal, with exaggerated deep tendon reflexes in both upper and lower limbs and both Planters extensor. His lower limb power was $4 / 5$ bilaterally and upper limb power was normal. The patient also had hyperesthesia over both the upper limbs.

On investigation the patient was found to have a cervical intradural extramedullary lesion compressing the spinal cord with a posterior connection to the skin in the form of a sinus (Figure 1A, 1B). The patient was admitted and after confirming no infection in the dermal tract, he was subjected to a laminectomy and tumor excision (Figure 1C). Post op his power stayed the same with reduction in tone. He was started on mobilatory rehabilitation and aggressive physiotherapy and made a complete recovery. The histopathology of the tumor excised showed dermal appendages with copious keratin and squamous epithelium suggestive of a dermoid.

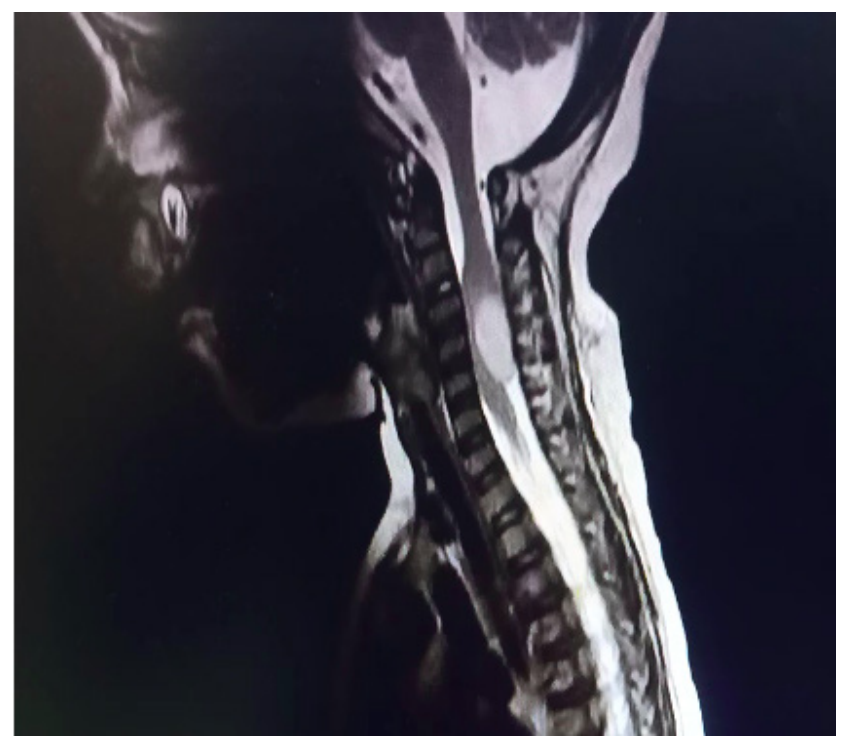

Figure IA T2 weighted sagittal MRI images of the cervical dermoid compressing the cord. 


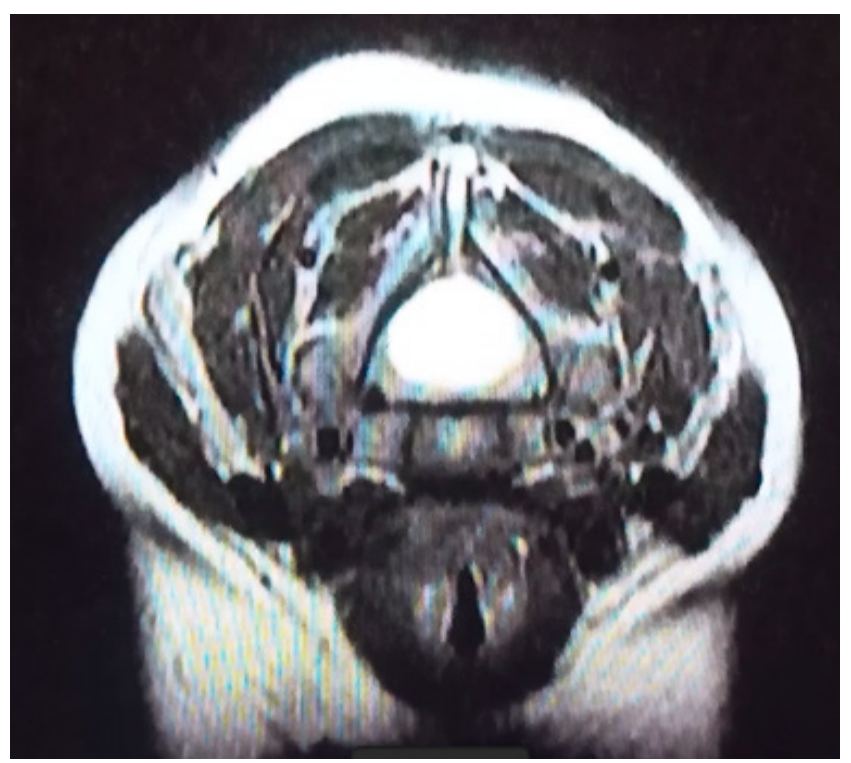

Figure IB T2 weighted axial MRI images showing the cord compression by the dermoid cyst.

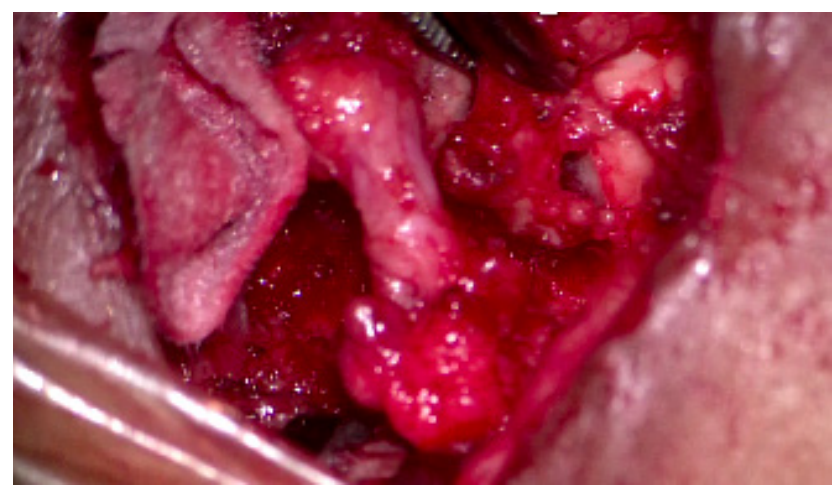

Figure IC Intraoperative image showing the dermal tract extending into the spine.

\section{Case 2}

A 4-year-old male child with delayed development milestones since infancy presented with history of frequent falls since last 5-6 months which gradually progressed to an inability to walk. He also complained of deteriorating hand grip. The relatives also noticed purulent discharge from back in the midline for 3 weeks which was not attended to appropriately. On examination, he was found to have a sinus over the upper back (D4 level) with purulent discharge from it. Neurological examination showed normal tone in all 4 limbs with power $3 / 5$ in all 4 limbs. Deep tendon reflexes were universally brisk and both planters were extensor. The child was also found to have significant cerebral malformations with included a large Dandy Walker Malformation present with significant cerebellar vermian agenesis, corpus callosal agenesis and polymicrogyria (Figure 2A).

The MRI of the spine showed a large intramedullary lesion at D4 to D6 causing cord dilation and obstructing the CSF gutters on either side (Figure 2B \& 2C). The patient was taken up for surgery after explaining the risks and consequences of such surgery. A D4 to D6 laminectomy was done and the lesion was found to be an intramedullary dermoid which was removed piecemeal under neuromonitoring cover. The (dural sinus was also removed along with the tumor. Postoperatively, the patient could walk with minimal support with power $4+/ 5$ in all limbs. Rehabilitation continued making him mobile after 3 months of intense physiotherapy.

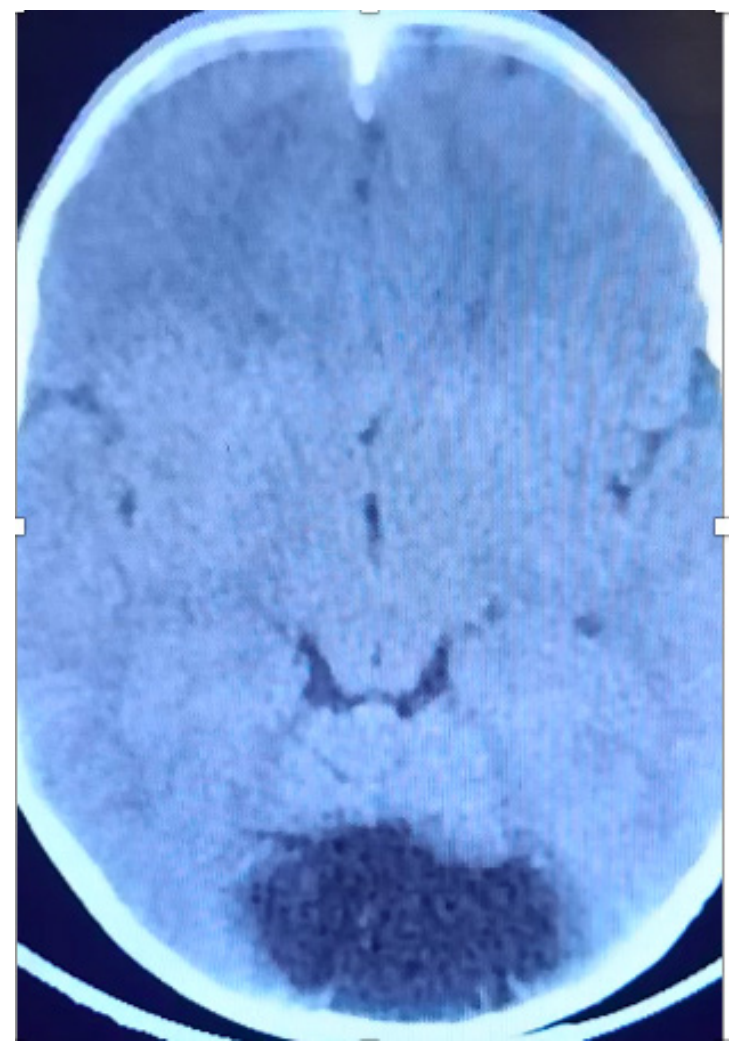

Figure 2A Vermian agenesis and a large CSF space in the posterior fossa suggestive of Dandy walker Malformation.

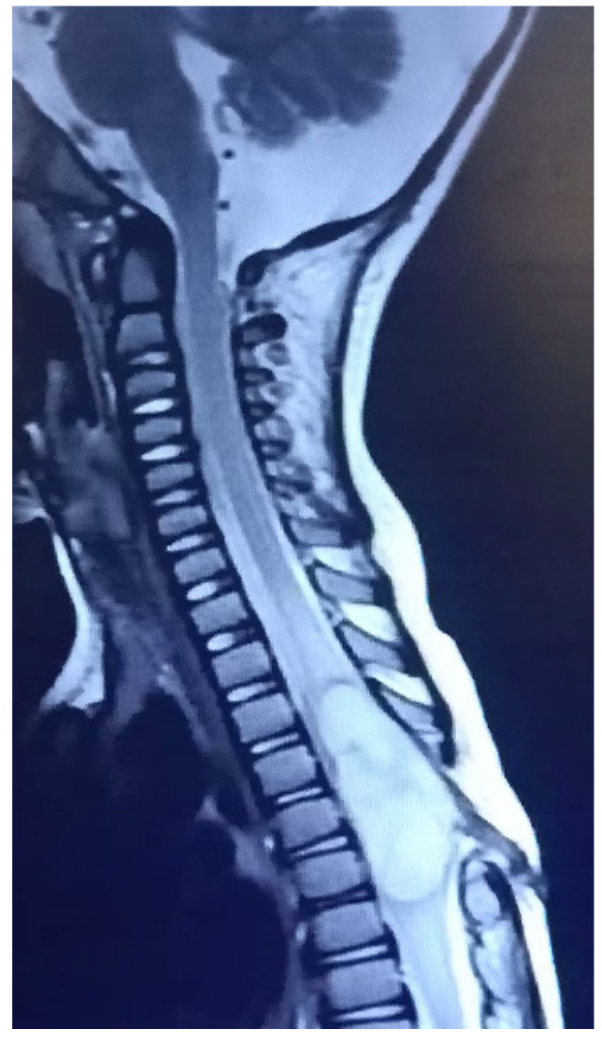

Figure 2B T2 weighted sagittal images showing the intramedullary lesion with enlargement of the cord. 


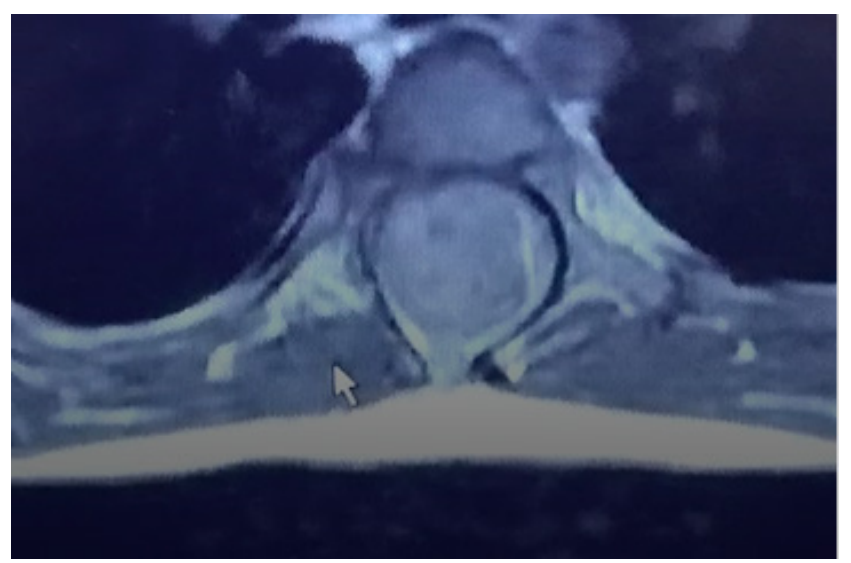

Figure 2C The intramedullary enlargement of the cord in a T2 weighted axial section.

\section{Case 3}

A 4-year-old female child Presented with complaints of weakness of both lower limbs for 3 years, progressive imbalance while walking and standing and difficulty in passing urine for 2 weeks. On examination she had wasting of the left lower limb, bilateral lower limb power $3 / 5$ with deep tendon reflexes brisk $(2+)$ in both lower limbs. Both plantars were flexor.

MRI of the spine showed a diastometamyelia at D12 with a large intradural extramedullary tumor compressing the conus medullaris along with tethering of the cord at L5 (Figure 3A, 3B). The child was taken up for a D12 to L1 laminectomy and correction of the split cord malformation and excision of the dermoid under neuromonitoring coupled with an L5 laminectomy and detethering of the cord. Both procedures were uneventful, and the patient improved well postsurgery. The hypertonia improved well, along with the weakness. The patient was able to walk with support after physiotherapy for 6 months.

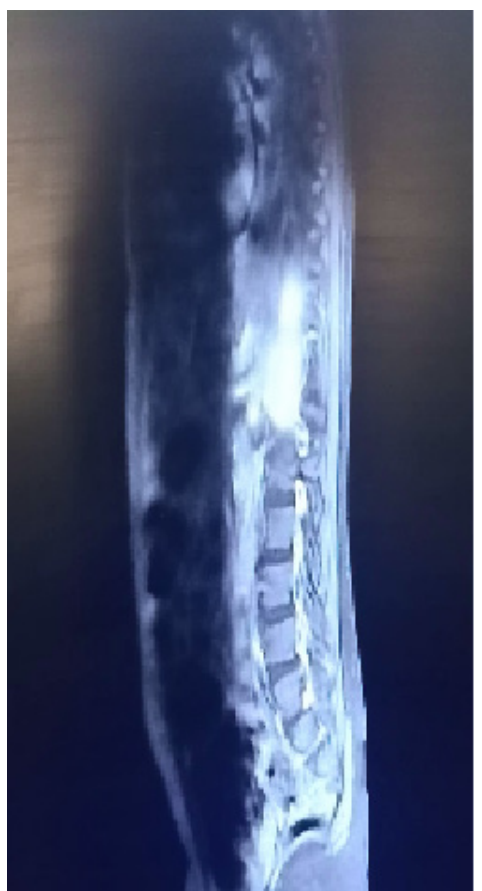

Figure 3A Sagittal T2 weighted MRI showing the split cord malformation and tethering of the cord.

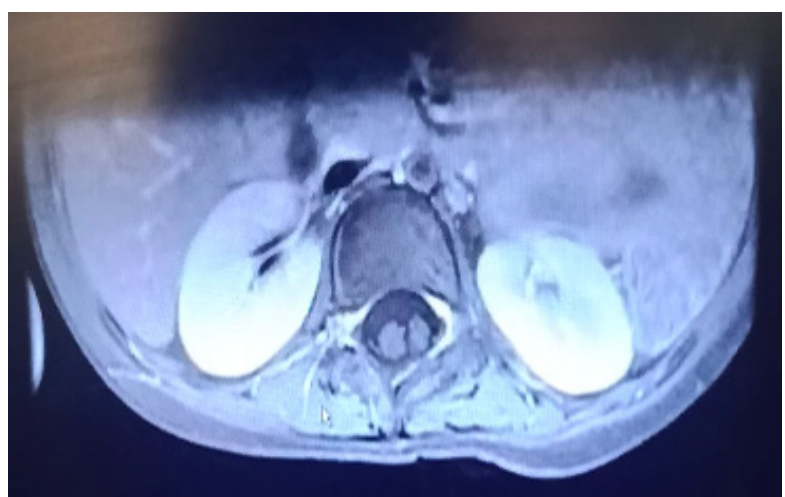

Figure 3B Axial T2 images showing the split cord separated by the membranous band.

\section{Case 4}

A 1 year 7 months old male child Presented with difficulty in walking for 6 months progressing gradually and now associated with incontinence for stool and urine for 1 month. For the last 2 days he had been having high grade intermittent fever with vomiting, rigours and chills. On examination he was found to be febrile with a temperature of 102-degree $\mathrm{F}$, with signs of sepsis. A dimple in the lumbosacral region in the midline with lead to a discharging sinus with pus. Neurological examination showed normal tone in all limbs with weakness in both lower limbs (3/5 symmetrical loss of power), with a lax anal sphincter tone. No Sensory deficit was present, but paraesthesia was present over both lower limbs.

MRI of the spine revealed a large intradural extramedullary lesion at L2 compressing the cauda equina. Tethering of the cord was noted at S2. Considerable inflammatory changes were seen around the lesion (Figure 4A, 4B). After appropriate antibiotic therapy for 21 days, the patient was taken up for surgery once inflammatory markers subsided. An L2 laminectomy was done and the lesion removed completely. Then an S2 laminectomy and detethering of cord was done along with excision of sinus tract. Post-surgery, the child was Ambulatory with minimal support with no new onset neurological deficit.

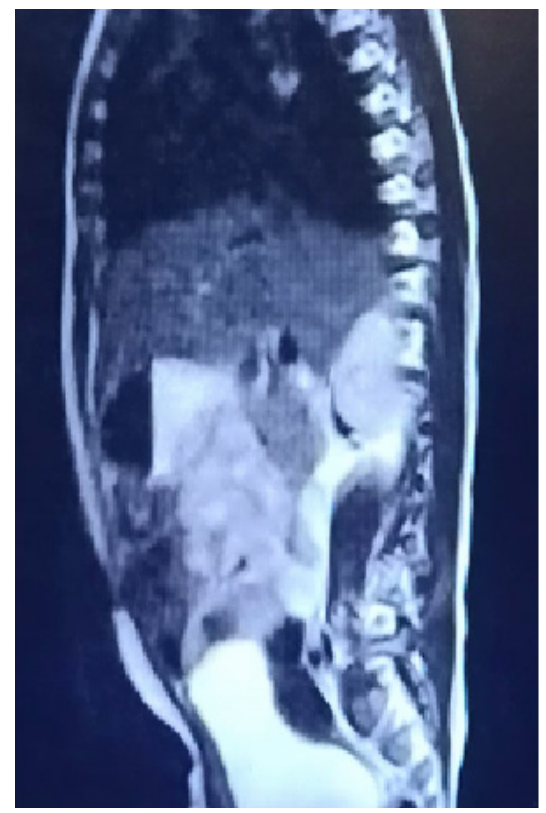

Figure 4A Sagittal MRI images showing a large dermoid compressing the cauda equina with significant inflammatory changes. 


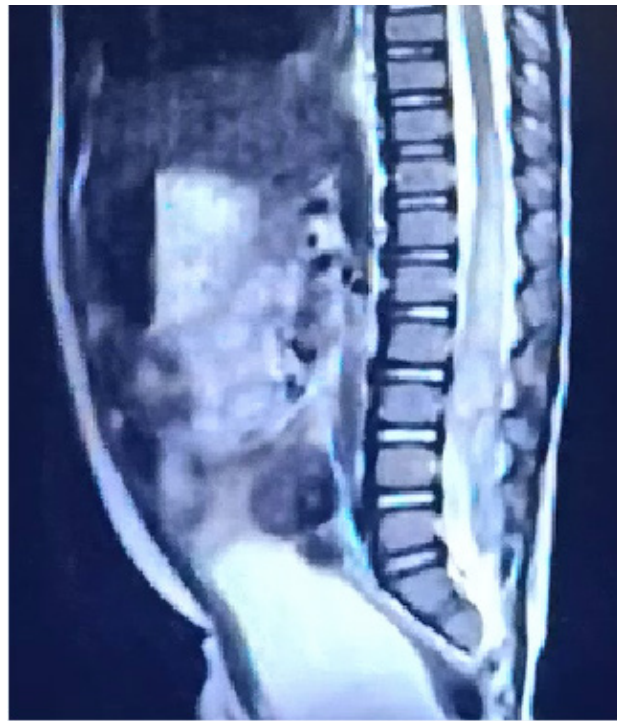

Figure 4B Sagittal T2 images showing the sinus tract communicating with the spinal canal.

\section{Discussion}

Also termed 'spinal cutaneous inclusion tumors', dermoids have been recognised as part of the dysraphism bouquet of lesions since decades. Intraspinal dermoid cysts are usually located in lumbar and thoracic regions and are usually associated with congenital spinal dysraphism and or dermal sinus tracts. ${ }^{2}$ Dermoids in the spine are rare, accounting for approximately $1.1 \%$ of all intraspinal tumors, and between $5 \%$ to $7 \%$ of all primary intradural tumors in the paediatric population. ${ }^{3}$ They are frequently associated with dermal sinuses which often get infected leading to meningitis and arachnoiditis. In children dermal sinus is the most common source of intramedullary abscess. ${ }^{3,4}$

Dermoid cysts are formed as a result of a defect in neural tube closure, which occurs between the $3^{\text {rd }}$ and $5^{\text {th }}$ gestational weeks. Cysts originates from the totipotent ectoderm inclusions, which could be either congenital or acquired. Ectodermal inclusions give rise to all the epidermal and dermal elements found in these cysts. During embryogenesis, the neural tube closes in the dorsal midline first in the cervical region and the closure then extending caudally to the lumbar and sacral regions. Hence, the lumbo-sacral region is the most common site for the dermoids in the spine. ${ }^{5,6}$

The causes and risk factors remain unclear. Standard risk factors for spinal dysraphism do not as per current literature predispose patients to develop dermoids, even though their association with dysraphism has been frequently mentioned. ${ }^{7}$ Dermoids are mostly congenital, where these inclusion cysts form early during embryonic development. Dermoids have been known to arise Iatrogenically as well due to the introduction of skin cells into the space around the spinal cord. ${ }^{8}$ This can happen as a result of surgery, most frequently surgery for closure of meningomyelocele or lipomeningeocoele which introduces epithelium into the spinal cord. Repeated lumbar punctures and spinal manipulations can also result in dermoids implantation and growth inside the spine. ${ }^{9}$

Six different forms of cutaneous markers have been described associated with dermoids. These tell-tale signs of spinal dysraphism are well documented signifying underlying midline embryonic disease. These include, Fatty lumbo-sacral mass in midline or parasagittal plane, hirsutism over the lumbar region, dermal sinus, haemangiomas, presence of a rudimentary tail and atretic meningocele. ${ }^{10}$ Radiology remains the basis of diagnosis and treatment planning. Plain $\mathrm{x}$ rays may demonstrate bony abnormalities of the spine including spina bifida and Scoliosis. CT scans can be utilized to delineate associated bony abnormalities especially split chord malformations, and spina bifida. Ultrasound is only useful in the infant spine and is useful primarily as a screening examination. ${ }^{11}$

MRI is the primary mode of diagnosis and is widely used for surgical planning apart from just diagnostics. The advantage of MRI is that one non-invasive modality manages to delineate multiple abnormalities in the neuraxis without disturbing their situation viz-a-viz the spinal cord. This investigation modality shows the location of the end of the spinal cord, the presence and location of tethering, associated anomalies such as dermal sinus tracts, split cord malformations, bony spinal defects, and other lesions such as lipomas and meningomyeloceles. ${ }^{12}$ Based on radiology, surgery can be planned, and prognosis can be deciphered so that an informed decision for surgery can be made by the whole team. ${ }^{13}$ Uroflowmetry while not always indicated can be used to assess the extent of the neurogenic bladder which often exists in longstanding cases, helping in prognostication of the urinary symptoms post-surgery. ${ }^{13}$

All symptomatic tethered cords must be surgically detethered as they will at some time produce deficits or pain. Other indications for surgery include dermal sinus tracts (blind or connecting with the spinal subdural space), any lesion causing myelopathy or radiculopathy, and finally to prevent rupture of dermoids which can lead to aseptic meningitis. The main goal of surgery is to stabilize neurologic progression, a proportion of patients improve as well as continue to progress despite detethering. ${ }^{14}$

After surgery, rehabilitation forms a major component of recovery of the patient. Limb physiotherapy and rehabilitation coupled with bladder control gradually improves. Longstanding compression of the cord leads to permanent disability which especially at a young age can be very severe. Prompt diagnosis and surgery followed by aggressive rehabilitation forms the core for ensuring good outcomes to patients of this rare lesion. ${ }^{15}$

\section{Conclusion}

Paediatric spinal dermoids are rare. The presence of a dermal sinus presents an added risk of Meningitis. Diagnosis is by radiology and treatment is surgical followed by rehabilitation. Lesions maybe seen alone or with other components of dysraphism. Long standing lesions may cause irreversible deficits (especially bladder and bowel). Hence early detection and prompt surgery are of the highest priority to reduce morbidity for patients.

\section{Acknowledgments}

None.

\section{Conflicts of interest}

The authors declare no conflicts of interest.

\section{Funding}

None.

\section{References}

1. Kukreja K, Manzano G, Ragheb J, et al. Differentiation between pediatric spinal arachnoid and epidermoid-dermoid cysts: is diffusion-weighted MRI useful?. Pediatric radiology. 2007;37(6):556-560. 
2. Wilson RE, Oleszek JL, Clayton GH. Pediatric spinal cord tumors and masses. The journal of spinal cord medicine. 2007;30(sup1):S15-S20.

3. van Aalst J, Hoekstra F, Beuls EA, et al. Intraspinal dermoid and epidermoid tumors: report of 18 cases and reappraisal of the literature. Pediatric neurosurgery. 2009;45(4):281-290.

4. Guo S, Xing Y. A review on five cases of intramedullary dermoid cyst. Child's Nervous System. 2014; 30(4):659-664.

5. Elton S, Oakes WJ. Dermal sinus tracts of the spine. Neurosurgical focus. 2001;10(1):1-4.

6. Shubha AM, Mohanty S, Das K, et al. Congenital inclusion tumours in spinal dysraphism. The Indian Journal of Pediatrics. 2010;77(2):167170.

7. Liu H, Zhang JN, Zhu T. Microsurgical treatment of spinal epidermoid and dermoid cysts in the lumbosacral region. Journal of Clinical Neuroscience. 2012;19(5):712-717.

8. Ackerman LL, Menezes AH. Spinal congenital dermal sinuses: a 30-year experience. Pediatrics. 2003;112(3):641-647.

9. Verma SK. Management problems of Infected Intra-spinal Dermoids. Journal of Pediatric Neurosciences. 2007;2(2).
10. Mishra SS, Panigrahi S. Thoracic congenital dermal sinus associated with intramedullary spinal dermoid cyst. Journal of pediatric neurosciences. 2014;9(1):30.

11. Jindal A. Mahapatra AK. Spinal congenital dermal sinus: an experience of 23 cases over 7 years. Neurology india. 2001;49(3):243.

12. Kumar A, Singh P, Jain P, et al. Intramedullary spinal epidermoid cyst of the cervicodorsal region: A rare entity. Journal of pediatric neurosciences. 2010;5(1):49

13. Shubha AM, Saleem KM, Deb M, et al. Intra Spinal Dermoids and Spinal Dysraphism. Journal of Indian Association of Pediatric Surgeons. $2007 ; 12(3)$

14. Baysefer A, Akay KM, Izci Y, et al. The clinical and surgical aspects of spinal tumors in children. Pediatric neurology. 2004;31(4):261-266.

15. Siller S, Egensperger R, Szelenyi A, et al. Intraspinal epidermoid and dermoid cysts - tumor resection with multimodal intraoperative neurophysiological monitoring and long-term outcome. Acta Neurochirurgica. 2020;1-9. 